\title{
El equinodermo Gogia granulosa (Echinodermata: Blastozoa) del Cámbrico temprano-medio en Sonora, México: paleoecología y paleogeografía
}

\author{
Blanca E. Buitrón Sánchez ${ }^{1}$, Francisco J. Cuen Romero², Alejandra Montijo González² \\ \& Sylvia Matilde Beresi ${ }^{3}$, \\ 1. Departamento de Paleontología, Instituto de Geología, Universidad Nacional Autónoma de México, Ciudad \\ Universitaria, 04510, CDMX, México; blancab@unam.mx \\ 2. Departamento de Geología, Universidad de Sonora, Blvd. Luis Encinas y Rosales, 83000, Hermosillo, Sonora, \\ México. \\ 3. Instituto Argentino de Nivología, Glaciología y Ciencias Ambientales, Consejo Nacional de Investigaciones \\ Científicas y Tecnológicas, Av. A. Ruiz Leal, Parque Gral. San Martín, 5500, Mendoza, Argentina.
}

Recibido 13-I-2017. Corregido 02-V-2017. Aceptado 16-VII-2017.

\begin{abstract}
The echinoderm Gogia granulosa (Echinodermata: Blastozoa) of early-mid Cambrian in Sonora, Mexico: paleoecology and paleogeography. Blastoids of Gogia granulosa Robison, 1965 and Gogia sp. have previously been reported from San Jose de Gracia, Sonora, Mexico. Here we report on the implications of their presence in limestone, shale, and sandstone from the lower-middle Cambrian of that site. The biotic association consists of blastoids, as well as trilobites (Onchocephalus, Bonnia, Bristolia, Olenellus), hyolithids (Hyolithes, Haplophrentis), sponges, algae and ichnofossils of echinoderms (Asteriacites, Asterosoma). The community suggests that the depositional environment was a carbonated platform of tropical, shallow sea, with well oxygenated water. The biota belonged to a large faunistic province that comprised parts of Canada, USA and Mexico (Panthalassic Ocean). Rev. Biol. Trop. 65(Suppl. 1): S160-S167. Epub 2017 November 01.
\end{abstract}

Key words: Blastozoa-Gogiida; Cambrian; Sonora; México.

Las investigaciones sobre el Paleozoico inferior marino de Sonora, cuyos estratos tienen una edad comprendida entre 540 millones de años a 359 millones de años, comenzaron con los estudios de King (1940) quien en el reconocimiento de la Sierra Madre Occidental citó la existencia de rocas del Cámbrico-Ordovícico en la región de Cobachi. Cooper \& Arellano (1946) y Cooper et al. (1952) trataron sobre la estratigrafía y el contenido biótico de la región de Caborca, ubicada al noroccidente del estado y dieron a conocer las primeras descripciones de algas calcáreas, arqueociatos, braquiópodos, moluscos y trilobites. Longoria \& Pérez (1979) publicaron el bosquejo geológico de los cerros Rajón y Chino en la región de Caborca. Baldis y Bordonaro (1981) establecieron la vinculación entre el Cámbrico del noroeste de México y el Cámbrico de la Precordillera Argentina.
Stewart et al. (1984) realizaron el estudio de las rocas del Proterozoico y Cámbrico de la región de Caborca. McMenamin (1985) informó sobre invertebrados de la Formación La Ciénega, NW Sonora. Rivera-Carranco (1988) publicó sobre las consideraciones paleoambientales de las formaciones cámbricas de Caborca. Almazán (1989) describió sobre el Cámbrico-Ordovícico de Arivechi, región centro oriental del Estado. Buitrón (1992) citó el contenido biótico de las rocas del Paleozoico inferior de Sonora y de otras localidades de México. Almazán et al. (2006) publicaron sobre una secuencia litoestratigráfica de plataforma del Ordovícico temprano de la región central de Sonora, México. Solís et al. (2013) escribieron un libro sobre las investigaciones de la diversidad de equinodermos en Latinoamérica y citaron la existencia de blastoideos-gógidos en Sonora, México. 
Particularmente, sobre la presencia de equinodermos del Cámbrico de México, Sprinkle (1973) mencionó que observó placas aisladas de blastoideos gógidos en Caborca, Sonora. Durham (1978) en sus estudios sobre equinodermos cámbricos, mencionó la posible presencia de blastoideos en la misma región. Nardin, et al. (2009) dieron a conocer la existencia de blastoideos de la especie Gogia granulosa Robison, 1965 y determinaron la edad Cámbrico inferior-medio de las rocas de San José de Gracia en la región central de Sonora. Cuen et al. (2016) hicieron el estudio detallado de la estratigrafía del cerro El Chihuarruita en esa región, y la relacionaron con la estratigrafía propuesta por Cooper, et al. (1952) para la región de Caborca situada al noroeste del estado. El presente estudio permitió la reevaluación de las unidades formacionales del cerro El Chihuarruita en la región de San José de Gracia, Sonora.

Los equinodermos blastoideos vivieron únicamente en el Paleozoico, con una antigüedad comprendida entre 540 a 252 millones de años. Su presencia fue más diversa en el Ordovícico, pero alcanzaron su mayor abundancia durante el Misisípico. Los fósiles de estos organismos son importantes índices estratigráficos, pues permiten conocer la edad relativa de las rocas que los contienen; además son la clave para entender algunos aspectos sobre la evolución temprana de los equinodermos. Los blastoideos se caracterizan por presentar la teca formada por placas organizadas en ciclos o irregularmente dispuestas, en la parte superior de la misma se encuentra la hidrospira $\mathrm{y}$ las braquiolas que tienen un origen axial y se formaron como extensiones ambulacrales que les permitieron filtrar agua con nutrientes (David et al., 2000; Zamora, 2010). El género Gogia Walcott, 1917 se clasifica en la familia Eocrinidae Jaekel, 1918, cuya teca presenta un número variable de placas poligonales organizadas en hileras alternantes, sin arreglo ordenado, con braquiolas largas, delgadas y biseriales (Ubaghs, 1967). Estas placas se encuentran rodeadas de poros suturales con función respiratoria (Walcott, 1917, p. 69, lám. 8, figs. 1a-b; Nardin et al. (2009, fig. 4).

El proceso de fosilización está influido por ciertos factores desfavorables entre ellos biológicos, físicos y químicos, que pudieron haber impedido la fosilización de los organismos o parte de ellos. La influencia subsecuente de procesos diagenéticos pudo haber causado la pérdida de ciertos componentes de la tanatocenosis o pudieron haber alterado la estructura de los fósiles como es el caso de los icnofósiles de equinodermos y de blastoideos gógidos de San José de Gracia, Sonora.

\section{MATERIALES Y MÉTODOS}

El material de blastoideos gógidos es escaso en la localidad de San José de Gracia y la mayoría se encontró desarticulado, también se hicieron secciones delgadas de las rocas para su análisis de microfacies y se encontraron fragmentos de las placas de la teca de Gogia e icnofósiles de equinodermos de las especies Asterosoma isp. y Asteriacites isp. El trabajo de campo se realizó con estricto control bioestratigráfico, basado en fósiles índice del Cámbrico, tales como blastoideos-gógidos, trilobites y braquiópodos. Este material proviene de rocas siliciclásticas de la Formación Proveedora y carbonatadas de la Formación Buelna.

Los ejemplares se encuentran depositados en la Colección de Paleontología "Emilio Almazán Vázquez" de la División de Ciencias Exactas y Naturales en la Universidad de Sonora (UNISON), Blvd. Luis Encinas y Rosales, 83000, Hermosillo, Sonora, México. Gogia granulosa (Formación Buelna) está registrada con los números de catálogo DPG-UNISON 321-06, DPG-UNISON 322-06 y Gogia sp. (Formación Proveedora) con el número de catálogo DPG-UNISON 231 a DPG-NISON-234. Las secciones delgadas de roca con placas de la teca de ejemplares de Gogia están registradas en la colección con los números SJG-65 a SJG67 (Formación Buelna).

Localidad geográfica. Las muestras de equinodermos estudiadas fueron recolectadas 
en afloramientos de la Formación Proveedora (540 millones de años) y de la Formación Buena (525 millones de años) del Cerro El Chihuarruita localizado en las cercanías del poblado de San José de Gracia, Municipio de Ures en la región central del estado de Sonora, México, con coordenadas $29^{\circ} 17^{\prime} 5^{\prime \prime} \mathrm{N}$ y $110^{\circ}$ 35 ' 3" W (Figura 1).

Contexto geológico y estratigráfico de Gogia granulosa Robison. La secuencia estratigráfica del cerro El Chihuarruita está conformada por rocas sedimentarias del Cámbrico inferior-medio distribuidas en las formaciones Proveedora, Buelna y Cerro Prieto, expuestas en la región de Caborca al oeste de Sonora y la nueva Formación El Gavilán (Figura 2). La Formación Proveedora consiste en arenisca y cuarzoarenita que contiene placas desarticuladas de la teca de Gogia asociadas con abundantes icnofósiles entre ellos, impresiones de equinodermos Asteriacites isp. y Asterosoma isp. (Seilacher, 2007; Buitrón et al., 2016). El contacto superior es concordante con la

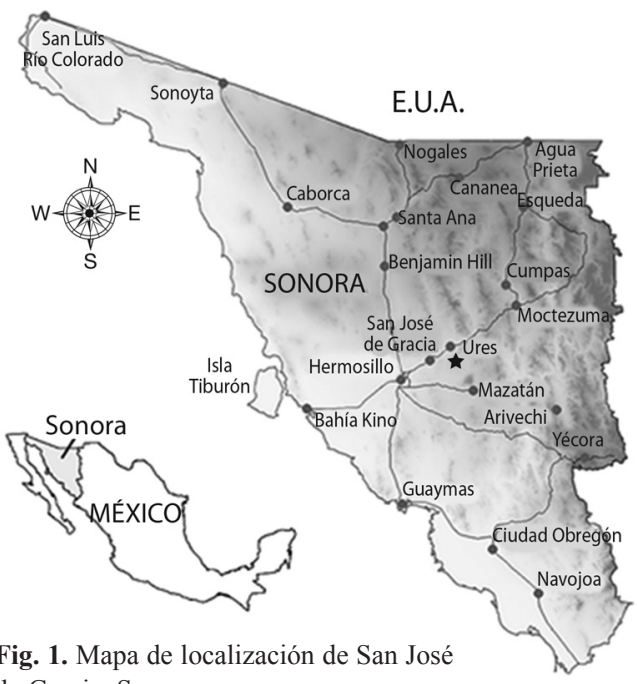
de Gracia, Sonora.

Fig. 1. Localization map showing the San José de Gracia, Sonora, México.

Formación Buelna conformada por caliza, lutita y caliza arenosa con fósiles de invertebrados, entre estos fragmentos de la teca y braquiolas de equinodermos blastoideos de la especie Gogia granulosa Robison cuya existencia

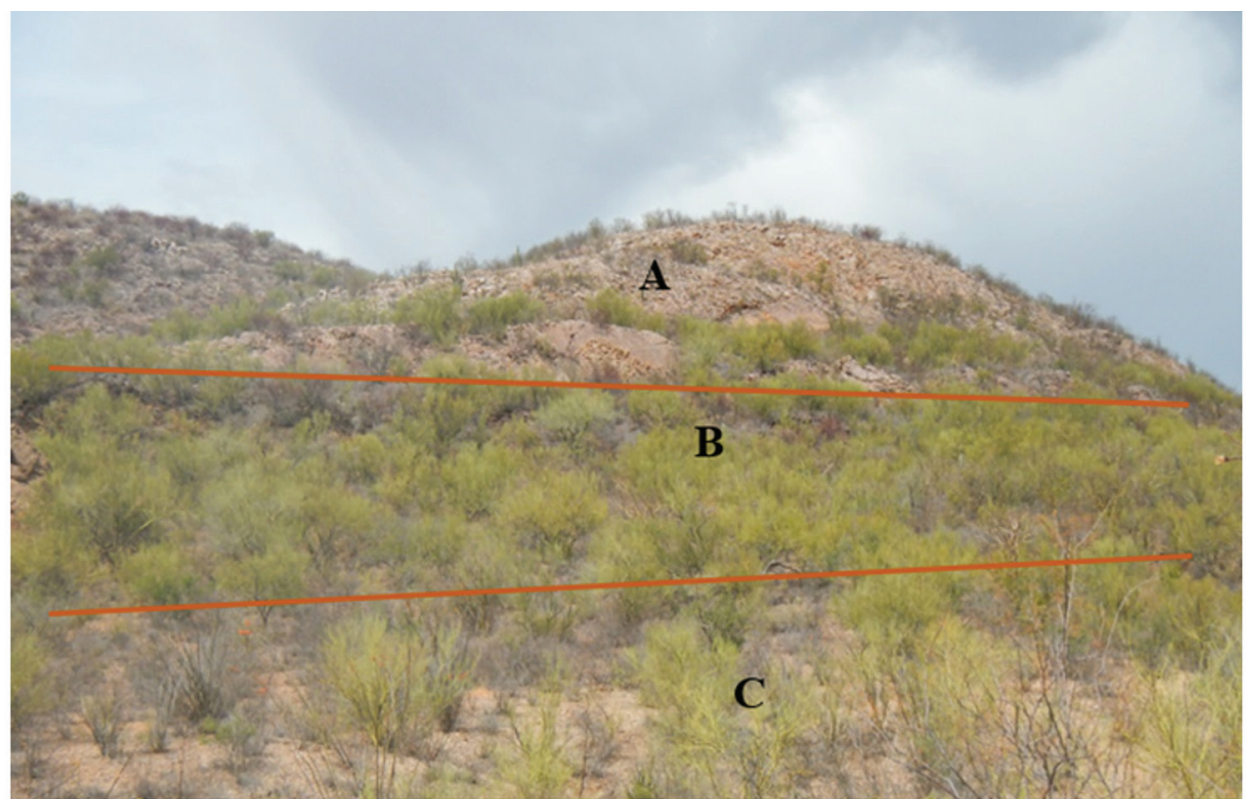

Fig. 2. Fotografía panorámica del cerro Chihuarruita que muestra los estratos de las Formaciones Proveedora (A), Buelna (B) y Gavilán (C).

Fig. 2. Panoramic photograph of El Chihuarruita hill showing the strata of the Proveedora (A), Buelna (B) and El Gavilán (C) formations. 
precisa la edad del Cámbrico inferior-medio (Nardin, et al., 2009), además, se encuentran algas oncolíticas, hiolítidos y trilobites. Posteriormente, aflora la Formación Cerro Prieto de caliza oolítica sin fósiles. Finalmente, la Formación El Gavilán está constituida por lutita intercalada con caliza y abundantes fósiles de esponjas, braquiópodos y trilobites que precisan la edad del Cámbrico medio (Cuen et al., 2016) y sobreyace en forma discordante por rocas volcánicas del Paleógeno (Figs. 2, 3).

\section{RESULTADOS}

En este trabajo se dan a conocer nuevos hallazgos de gógidos procedentes

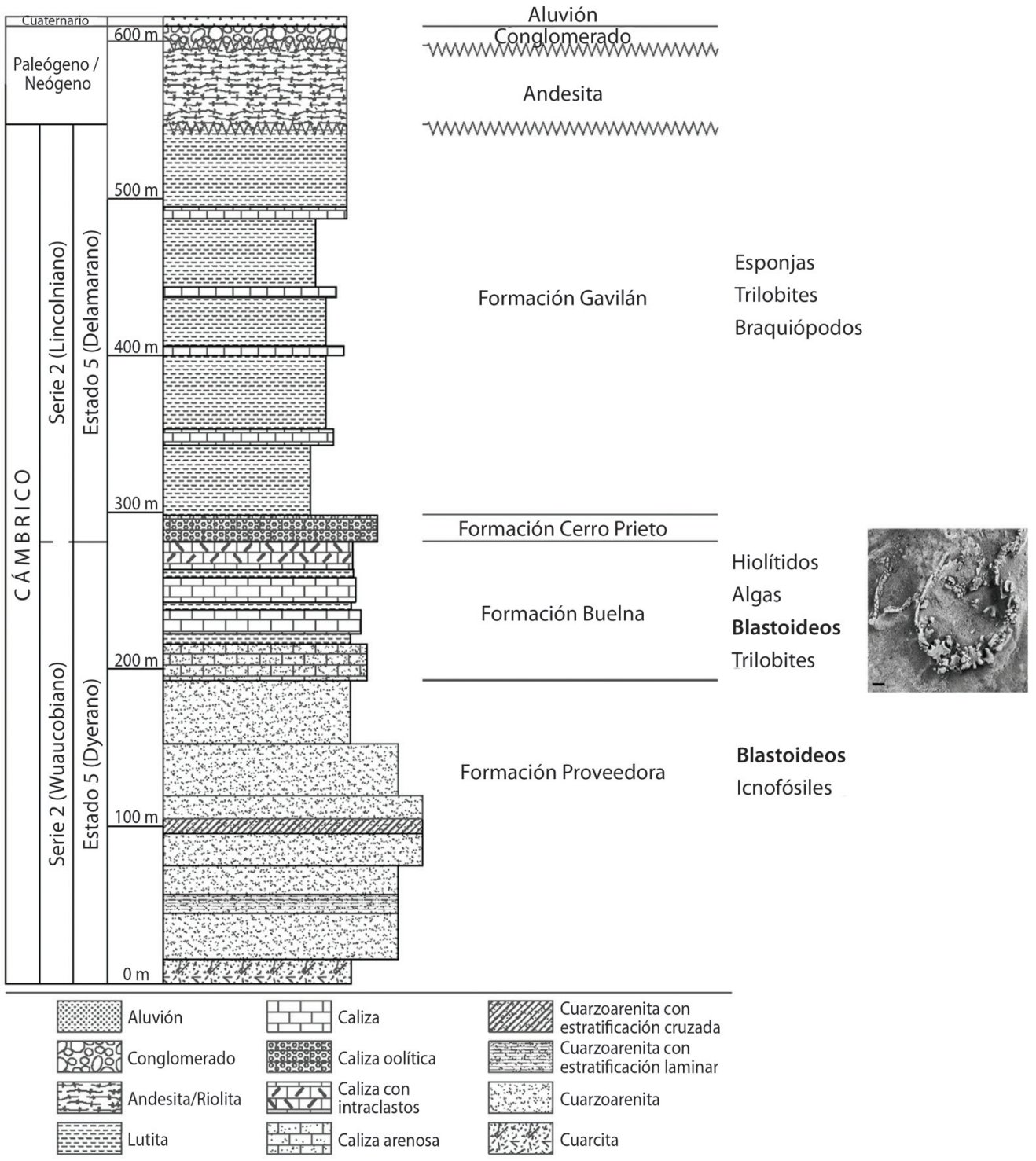

Fig. 3. Columna estratigráfica que muestra la posición de Gogia granulosa Robison, 1965 y de los icnofósiles Asteriacites isp. y Asterosoma isp.

Fig. 3. Stratigraphic column showing the position of Gogia granulosa Robison, 1965, and of the ichnofossils Asteriacites isp. and Asterosoma isp. 
de afloramientos de las Formaciones Proveedora (Fig. 4 C) y Buelna en San José de Gracia. En el análisis de las microfacies de la Formación Buelna se reconocieron placas de Gogia sp. (Fig. 5 A, B, C). Asimismo, se encontraron asociados icnofósiles de equinodermos identificados con Asterosoma isp. y Asteriacites isp. en las rocas de esta formación (Fig. 6 A, B, C).

Consideraciones paleoecológicas. En el Cámbrico comienza la mayoría de los phyla que existen hoy en día, presuntamente originados en la "Explosión de vida", sin embargo, hay episodios de extinción masiva (Liñán \& Gámez-Vintaned, 1999). La vida durante este período se desarrolló principalmente en ambiente marino, con organismos de cuerpo blando que a través del tiempo formaron conchas como protección a los depredadores (McAlester, 1973; Laporte, 1974). Con relación a los gógidos, se trata de organismos epifaunales estacionales que se alimentaban de manera suspensívora y que, en este caso, se encontraron en estratos calcáreos y arcillosos que denotan energía baja a moderada, con aportes repentinos de materiales provenientes
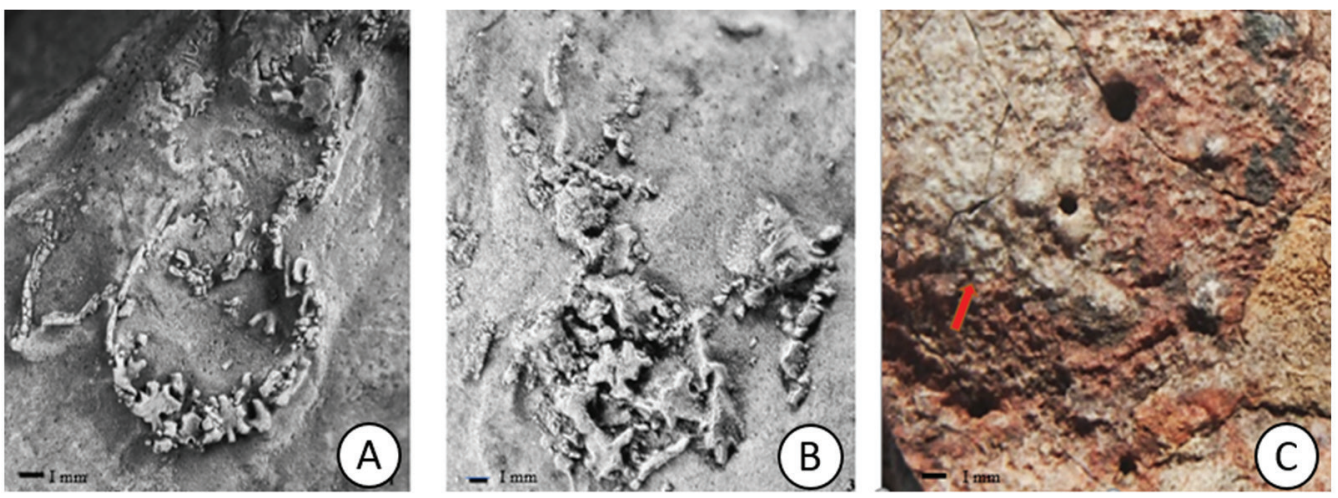

Fig. 4. Gogia granulosa Robison, 1965. A) Ejemplar DPG-USON321-06 y B) DPG-USON322-06. Fotografías tomadas de Nardin, et al., (2009). C. Ejemplar USON-DG-231 que muestra placas disociadas de la teca de eocrinoideos-gógidos (marcadas con una flecha) y orificios de Arenicolites isp. (Formación Proveedora).

Fig. 4. Gogia granulosa Robison, 1965. A) Sample DPG-USON321-06 and B) DPG-USON322-06. Scale $=1 \mathrm{~mm}$. Photographs taken from Nardin, et al. (2009). Specimen USON-DG-231 showing isolated plates eocrinoid-gógidos (marked with an arrow) and holes generated by icnofossils from Arenicolites isp. in the Proveedora Formation.
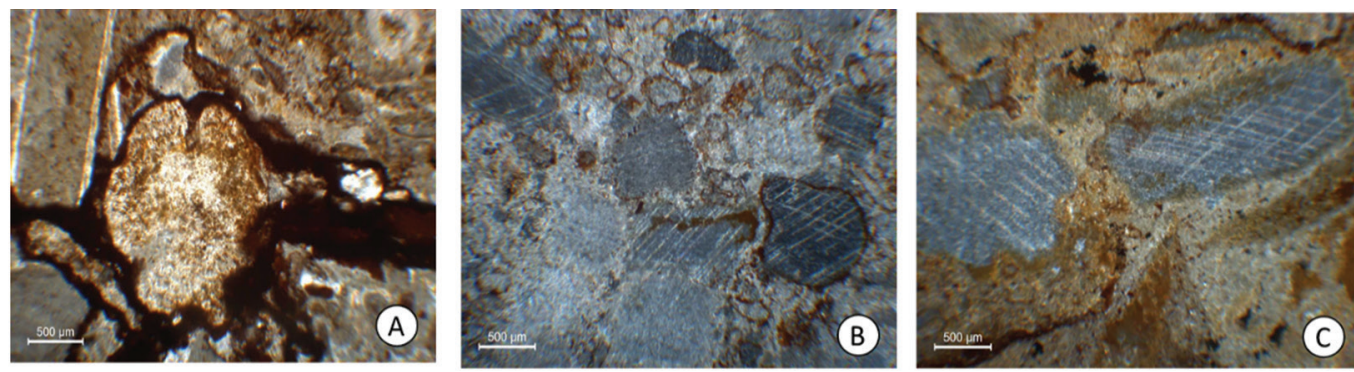

Fig. 5. Secciones delgadas de la Formacion Buelna. A Caliza con intraclastos y placa de Gogia sp. recristalizada por esparita, en una matriz de micrita y abundante óxido de fierro (No. SJG-65). B. Caliza con placas de Gogia sp, delimitadas por óxido de fierro, en una matriz de micrita (SJG-66). C. Caliza con placas aisladas de Gogia sp, de coloración gris y óxidos de fierro en una matriz de micrita (SJG-67).

Fig. 5. Thin section of the Buelna Formation. A Limestone with intraclasts and plates of Gogia sp. recrystallized from esparite, in a matrix of micrite and abundant iron oxide. B. Limestone with plates of Gogia sp, delimited by iron oxide, in a micrite matrix. C. Limestone with isolated plates of Gogia sp, with reddish coloration due to the iron oxides in a matrix of micrita. 

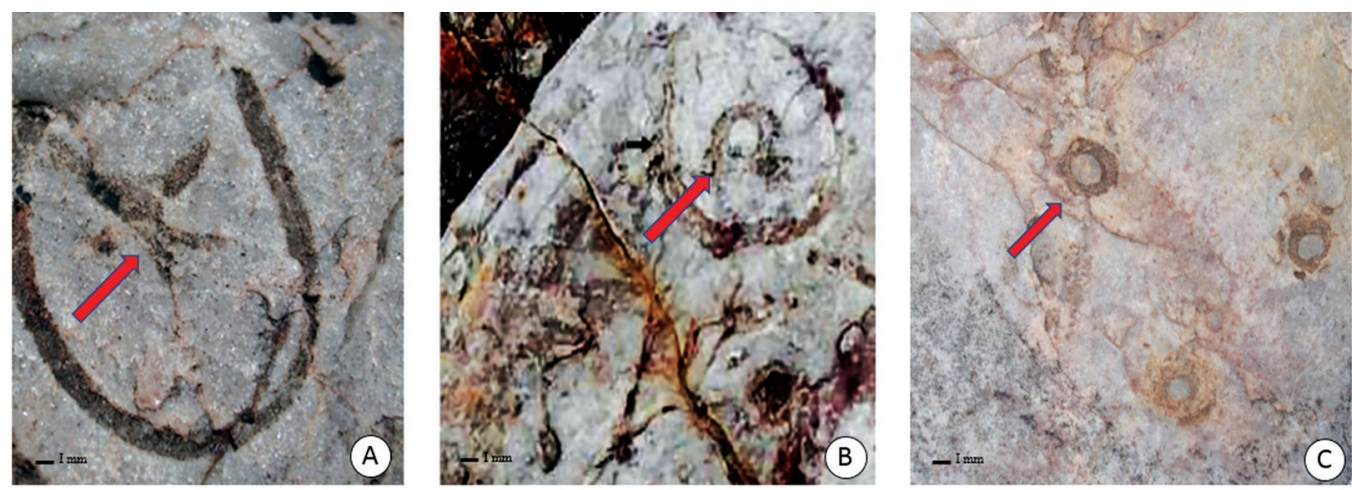

Fig. 6. Las fotografias corresponden a cuarzo arenita con icnofósiles A. La roca muestra la forma estelar del Icnofósil Asteriacites isp. (No. SON-DG-232). B. y C. Roca con icnfosiles del equinodermo Asterosoma isp. (Nos. USON-DG-233, USON-DG-234).

Fig. 6. The photographs correspond to Sandstone quartz with icnofosiles A. The rock shows the stellate form of Icnofósil Asteriacites isp. (No. SON-DG-232). B and C. Rock with ichnfosiles of the echinoderm Asterosoma isp (Nos. USONDG-233, USON-DG-234).

del exterior. Las capas calcáreas en las que se presentan estos organismos tienen inclusiones de fragmentos de roca, depositados por acción del oleaje y posiblemente removidos de bancos arrecifales cercanos. Estos organismos eran tolerantes a variaciones en las condiciones del medio por lo que pudieron haberse establecido cerca de zonas someras a muy someras como lo sugiere la presencia de icnofósiles de los equinodermos Asteriacites, Asterosoma y trilobites (Buitrón et al., 2016; Cuen et al., 2016). Otros organismos que formaron la biota del Cámbrico de San José de Gracia, fueron las algas oncolíticas (Beraldi et al., 2013), esponjas (Cuen et al., 2013 ), hiolítidos de las especies Hyolithes sonora Lochman, Haploprentis reesei Walcott (Buitrón, et al., 2017) trilobites de los géneros Onchocephalus, Bonnia, Bristolia, Olenellus (Cuen et al., 2016) y braquiópodos, habitantes del bentos marino, los cuales se asocian a condiciones paleoambientales correspondientes a una plataforma detrítica interna con baja a alta energía (Mount, 1980, Zamora et al., 2010).

Consideraciones paleogeográficas. La presencia de Gogia sp. y Gogia granulosa en Sonora central, NW México, permitió establecer relaciones paleogeográficas con Alberta en Canadá, California, Nevada, Utah y Idaho en los Estados Unidos de Norteamérica; Francia,
Corea del Norte y Australia (Nardin et al., 2009). También en España se han reportado gógidos en las Cadenas Ibéricas, Sierras de la Demanda, Zonas Cantábrica y de Ossa Morena (Zamora, 2010, 2011).

Reconstrucciones paleogeográficas de Norteamérica sugieren que el margen occidental se encontraba localizado en los trópicos, orientado este-oeste durante el Cámbrico, como consecuencia de esta ubicación, la fauna de San José de Gracias es única, entre ellos los gógidos, braquiópodos, hiolítidos y trilobites que demuestra existió una gran provincia, la cual incluyó varias localidades en Sonora, México (Caborca, San José de Gracia, Mazatán, Arivechi), en Canadá (Alberta) y en Estados Unidos de América (Nevada, Idaho, Utah, California) que formaban parte del Océano Pantalásico (Figura 7).

La asociación biótica de las formaciones Proveedora y Buelna, está conformada por blastoideos gógidos de las especies Gogia granulosa Robison, 1965 y Gogia sp., algas oncolíticas, icnofósiles de equinodermos (Asteriacites, Asterosoma), trilobites (Onchocephalus, Bonnia, Bristolia, Olenellus), hiolítidos (Hyolithes, Haplophrentis) y esponjas que son considerados fósiles índices del Cámbrico inferior y medio, que permitieron la reevaluación de las unidades formacionales del cerro 


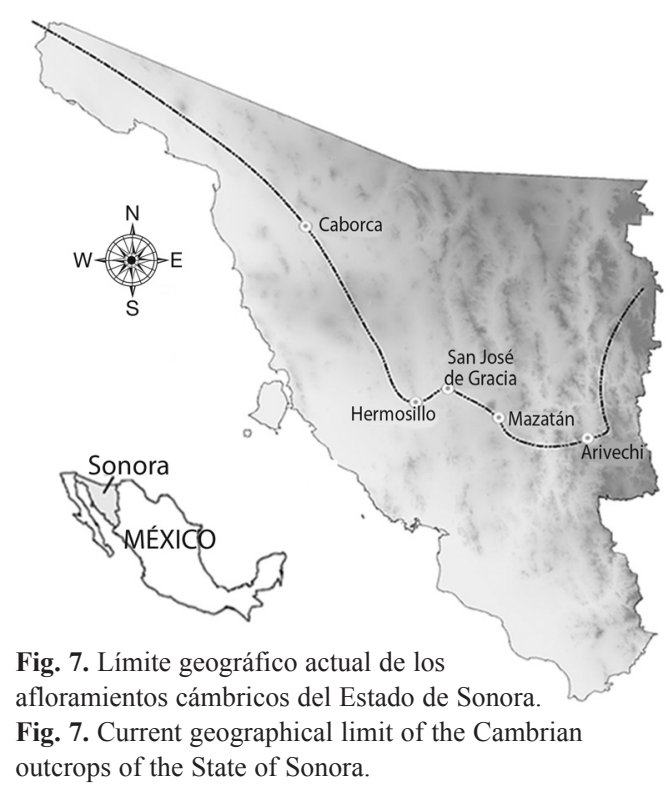

El Chihuarruita en la región de San José de Gracia, Sonora central.

Los equinodermos-blastoideos de la especie Gogia granulosa y la fauna asociada presentes en los estratos de la Formación Buelna, sugieren una serie de variaciones ambientales que incluyen registros de plataforma carbonatada de mar tropical, somero, con agua bien oxigenada, que prevaleció durante el Cámbrico en la región de San José de Gracia, Sonora.

La distribución de los blastoideos gógidos y la biota asociada denota que existió una amplia provincia faunística que comprendió en Canadá a la Columbia Británica, en los Estados Unidos de Norteamérica a Nevada, Idaho, Utah y California y en México a las regiones de Caborca, San José de Gracia, Mazatán y Arivechi, Sonora, las cuales formaban parte del Océano Pantalásico.

\section{AGRADECIMIENTOS}

Los autores agradecen a Silvia Martínez, jefa del Departamento de Geología, Universidad de Sonora, el apoyo logístico para la realización de este trabajo. La investigación se llevó a cabo en el marco de los proyectos CONACyT No. 165826; ECOS-Francia;
CONACyT-México No. M13U01 "Evolución de los Ecosistemas del Paleozoico de México" y CONACyT No. 235970 "La Revolución del Cámbrico y la gran radiación del Ordovícico en el norte de México y Oaxaca". Se agradece a María Esther Diupotex Chong del Laboratorio de Taxonomía y Ecología de Equinodermos ICML UNAM la preparación del nuevo material de estudio.

\section{RESUMEN}

Blastoideos de Gogia granulosa Robison, 1965 y Gogia sp. han sido previamente reportados para San José de Gracia, Sonora, México. Aquí nosotros reportamos de las implicaciones de su presencia en piedra caliza, pizarra y arenisca para el Cámbrico bajo-medio de este sitio. La asociación biótica consistío de blastoideos, así como de trilobites Onchocephalus, Bonnia, Bristolia, Olenellus), hyolitidos (Hyolithes, Haplophrentis), esponjas, algas e ignofósiles de equinodermos (Asteriacites, Asterosoma). La comunidad sugiere que el ambiente de deposición fue una plataforma carbonatada tropical, de mar somero, con aguas bien oxigenadas. La biota perteneció a una gran provincia faunística que comprendió partes de Canadá, USA y México (Océano Panthalasico).

Palabras claves: Blastozoa-Gogiida; Cámbrico; Sonora; México.

\section{REFERENCIAS}

Almazán, V. E. (1989). El Cámbrico-Ordovícico de Arivechi, en la región centro-oriental del estado de Sonora. Universidad Nacional Autónoma de México, Instituto de Geología, Revista, 8, 58-66.

Almazán, V.E., Buitrón-Sánchez, B.E. \& Franco-Vega, O. (2006). Formación Pozo Nuevo: una secuencia litoestratigráfica de plataforma del Ordovícico Temprano de la región central de Sonora, México. Revista Mexicana de Ciencias Geológicas, 23, 23-38.

Baldis B.A.J. \& Bordonaro O.L. (1981). Vinculación entre el Cámbrico del noroeste de México y la Precordillera Argentina. Porto Alegre, Brasil, II Congreso Latinoamericano de Paleontología, Annals, 1, 1-10.

Beraldi, C.H., Buitrón, B.E., Cuén R. F., Montijo A. (2013). Oncolitos del Cámbrico Inferior de la región de San José de Gracia, Sonora. VIII Congreso Latinoamericano de Paleontología y XIII Congreso Mexicana de Paleontología, Guanajuato 23-27 de septiembre. Resúmenes

Buitrón, B.E. (1992). Las rocas sedimentarias marinas del Paleozoico Inferior de México y su contenido biótico. 
In J.G. Gutiérrez Marco, J. Saavedra \& I. Rábano (Eds.) Paleozoico Inferior de Ibero-América. (193201 pp.) España: Universidad de Extremadura.

Buitrón, B.E., Corona-González, N., Cuen-Romero, F., Palafox-Reyes, J.J. \& Ramírez-Guerrero, G. (2016). Icnofósiles del Cámbrico Inferior de San José de Gracia, Sonora. Paleontología Mexicana, 5, 33-40.

Buitrón- Sánchez, B., Cuen-Romero, F., Huerta-Ruiz, A., Montijo-González, A. (2017). Hiolítidos del Cámbrico (Hyolitha) de San José de Gracia, Sonora, México. Consideraciones estratigráficas y paleogeográficas. Paleontología Mexicana 6, 1, 25-34.

Cooper, G.A. \& Arellano, A. (1946). Stratigraphy near Caborca, northwestern México, Sonora. American Association of Petroleum Geologists, 30, 606-610.

Cooper, G. A., Arellano, A., Johnson, J., Okulitch, J., Stoyanow, A. \& Lochman, C. (1952). Cambrian stratigraphy and paleontology near Caborca, northwestern Sonora, México. Smithsonian Miscellaneous Collections, 119, 1-178.

Cuen, F., Beresi, M., Montijo, A., Buitrón, B., Minjarez, S., De La O, M. \& Palafox, J.J. (2013). Chancelloria Walcott, 1920 y Reticulosa Reid, 1958 del Cámbrico Medio de San José de Gracia, Sonora, México. Boletín de la Sociedad Geológica Mexicana, 65, 581-590.

Cuen, R.F., Valdez-Holguín, J.E., Buitrón, B.E., Monreal, S.R., Sundberg, F., Montijo, G.A. \& Minjarez, S. I. (2016). Cambrian, Stratigraphy of San José de Gracia, Sonora, México: El Gavilán Formation a new lithostratigraphic unit of middle Cambrian open shelf environment, Boletín de la Sociedad Geológica Mexicana, 68, 429-441.

David, B, Lefebvre, B, Mooi, R, Parsley, RL. (2000). Are homalozoans echinoderms? An answer from the extraxial-axial theory. Paleobiology, 26, 529555, doi: 10.1666/0094-8373(2000)026<0529: AHEAAF $>2.0 . \mathrm{CO} ; 2$

Durham, J. W. (1978). A Lower Cambrian eocrinoid. Journal of Paleontology 52, 195-199.

Hallam, A. (1973). Atlas of paleobiogeography. Amsterdam: Elsevier. 531 p.

King, R.E. (1940). Pre-Tertiary history of the Sierra Madre Occidental of Sonora and Chihuahua and some adjacent parts of central Sonora, México. Sexto Congreso Científico, Proceedings, 1, 217-222.

Laporte, L.F. (1974). Los ambientes antiguos. Fundamentos de las Ciencias de la Tierra. España: Omega. 115 p.

Liñán, E. \& Gámez-Vintaned, J. (1999). La radiación Cámbrica: ¿Explosión de biodiversidad o de fosilización? Boletín de la Sociedad Entomológica Aragonesa, $26,133-143$.

Longoria, J. \& Pérez, V. (1979). Bosquejo geológico de los cerros Chino y Rajón, cuadrángulo Piquito-La
Primavera, NW de Sonora. Boletín del Departamento de Geología de la Universidad de Sonora, 1, 119-144.

McAlester, A.L. (1973). La historia de la vida. Fundamentos de las Ciencias de la Tierra. España: Omega. $151 \mathrm{p}$.

McMenamin, M.A.S. (1985). Basal Cambrian small shelly fossils from the La Ciénega Formation, northwestern Sonora, México. Journal of Paleontology, $59,1414-1425$.

Mount, J.D. (1980). Characteristics of Early Cambrian faunas from eastern San Bernardino County, California. Southern California Paleontological Society, Special Publication, 2, 19-29.

Nardin, E., Almazán-Vázquez, E. \& Buitrón-Sánchez, B.E. (2009). First report of Gogia (Eocrinoidea, Echinodermata) from the Early-Middle Cambrian of Sonora (México), with bioestratigraphical and paleoecological comments. Geobios, 42, 233-242.

Rivera-Carranco, E. (1988). Condiciones paleoambientales de depósito de las formaciones cámbricas del área de Caborca, Sonora Noroccidental. Universidad Nacional Autónoma de México, Instituto de Geología, Revista, 7, 22-27.

Seilacher, A. (2007). Trace Fossils Analysis. Berlin: Springer-Verlag. $226 \mathrm{p}$.

Solís M., F., Alvarado J. J., Buitrón, B.E., Durán, A., 2013. Echinoderms research and diversity in Latin America, Chapter 2: "The Echinoderms of Mexico: Biodiversity, distribution and current state of knowledge" Capítulo 2. Primera edición, Reino Unido, ISBN 978-3-64-200051-9,

Sprinkle, J. (1973). Morphology and evolution of blastozoan echinoderms. Harvard University, Museum of Comparative Zoology Special Publications, 1-284.

Stewart, J., McMenamin, A. \& Morales-Ramírez, J. (1984). Upper Proterozoic and Cambrian Rocks in the Caborca Region, Sonora, Mexico - Physical Stratigraphy, Biostratigraphy, Paleocurrent Studies, and Regional relations. United States Geological Survey Professional Paper, 1309, 1-36.

Ubaghs, G. (1967). Echinodermes nouveauxdu Cambrien moyende la Montaine Noire (France). Annales de Paléontologie 73, 1-27.

Walcott, C.D. (1917). Cambrian geology and paleontology, fauna of the Mount Whyte Formation. Smithsonian Miscellaneous Collections 67, 61-114.

Zamora, S. (2010). Equinodermos del Cámbrico: Planes corporales, paleoecología y registro fósil en España. Cidaris 30, VIII EJIP, 37-47.

Zamora S. (2011). Equinodermos del Cámbrico de España: situación actual de las investigaciones y perspectivas futuras. Estudios Geológicos, 67,1, 59-81. ISSN: 0367-0449, doi:10.3989/egeol.40240.121 